\title{
Post-renal acute renal failure due to a huge bladder stone
}

\author{
Orcun Celik, Tufan Suelozgen, Salih Budak, Yusuf Ozlem Ilbey \\ Tepecik Educational and Research Hospital Urology Department, 35140, Izmir, Turkey
}

\begin{abstract}
Summary A 63-year old male was referred to our emergency unit due to acute renal failure. The level of serum renal function tests levels, blood urea nitrogen (BUN)/creatinine, were $63 \mathrm{mmol} / \mathrm{L} / 848$ $\mu \mathrm{mol} / \mathrm{L}$. CT (Computarised Tomography) scan showed a huge bladder stone $(5 \mathrm{~cm} \times 6 \mathrm{~cm} \times 5 \mathrm{~cm})$ with increased bladder wall thickness. Post-renal acute renal failure due to bilateral ureterohydronephrosis was diagnosed. The huge bladder stone was considered to be the cause of ureterohydronephrosis and renal failure. The patient was catheterised and received haemodialysis immediately. He received haemodialysis four times during ten days of hospitalization and the level of serum renal function tests levels (BUN/ creatinine) decreased $18 \mathrm{mmol} / \mathrm{L} / 123$ $\mu \mathrm{mol} / \mathrm{L}$. After improvement of renal function, we performed cystoscopy that demonstrated normal prostatic urethra and bladder neck and bilaterally normal ureteral orifices. Bladder wall was roughly trabeculated and Bladder outlet was completely obstructed by a huge bladder stone. After cystoscopy open, cystolithotomy was performed to remove calcium phosphate and magnesium ammonium phosphate stone weighing $200 \mathrm{~g}$ removed. Four days after operation the patient was discharged uneventfully and urethral catheter was removed on the seventh day. Post-renal acute renal failure due to large bladder stones is rare in literature. According to the our knowledge; early diagnosis of the stone avoid growth to large size and prevent renal failure.
\end{abstract}

KEY WORDS: Bladder calculus; Acute renal failure; Open cystolithotomy; Ureterohydronephrosis.

Submitted 28 April 2014; Accepted 31 May 2014

\section{INTRODUCTION}

The pathogenesis of bladder stones has not been well studied (1). Bladder stones account for 5\% of urinary stones and occur in association with urinary tract infection, foreign bodies and neurogenic voiding dysfunction (2). In our recent urology practice, massive bladder stone is a rare entity. The association between bladder calculi and urinary stasis is not completely clear. Unlike studies of upper urinary tract stone disease, factors contributing to the pathogenesis of bladder calculi have not been well explored. Although, bladder stones are usually associated to ureteral or renal stones, they may occasionally occur alone in absence of stones of the supravesical tract, as in our case (3).

\section{Case report}

We present a 63-year-old man referred to our emergency unit for recurrent urinary tract infection, lower abdominal pain, oliguria and weight loss since six months. Hepresented lower urinary tract symptoms including weak urine stream, urgency, urinary frequency, voiding difficulty and urinary intermittency. At physical examination he was anorexic and weak. Blood urea nitrogen (BUN) and serum creatinine levels were $63 \mathrm{mmol} / \mathrm{L}$ and $848 \mu \mathrm{mol} / \mathrm{L}$ respectively. CT (computerised tomography) scan showed a huge bladder stone $(6 \mathrm{~cm} \times 6 \mathrm{~cm} \times 5 \mathrm{~cm})$ with increased bladder wall thickness and bilateral ureterohydronephrosis (Figures 1-2).

Neurologic examination was normal, therefore urodynamic evaluation was not indicated pre and post-operatively. Haemodialysis and urethral catheterization improved renal function, whereas we simultaneously treated urinary tract infection with intravenous antibiotics during ten days hospitalization. We planned cystoscopy and open cystolithotomy.

Figures 1.

The huge pelvic calculi seen in CT scan.

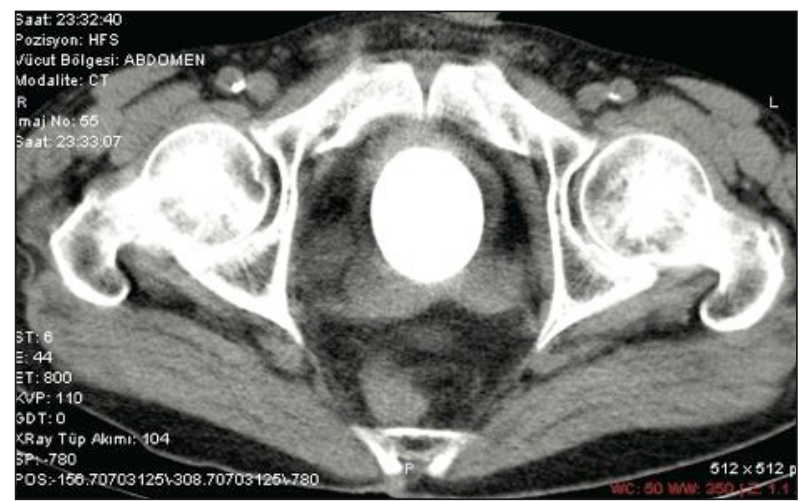


Figures 2.

Bilateral ureterohydronephrosis that cause of acute renal failure seen in CT scan.

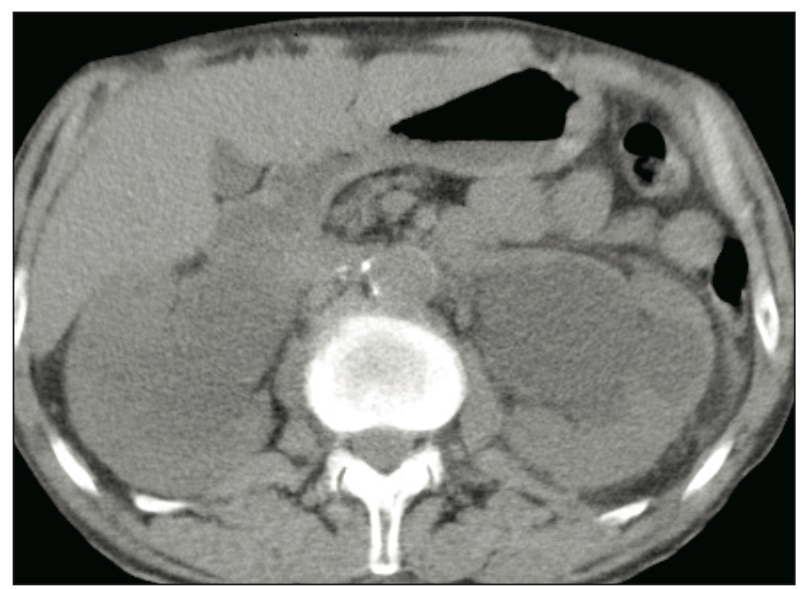

Figures 3.

A $6 \times 5 \mathrm{~cm}$ bladder stone was extracted by open cystolithotomy.

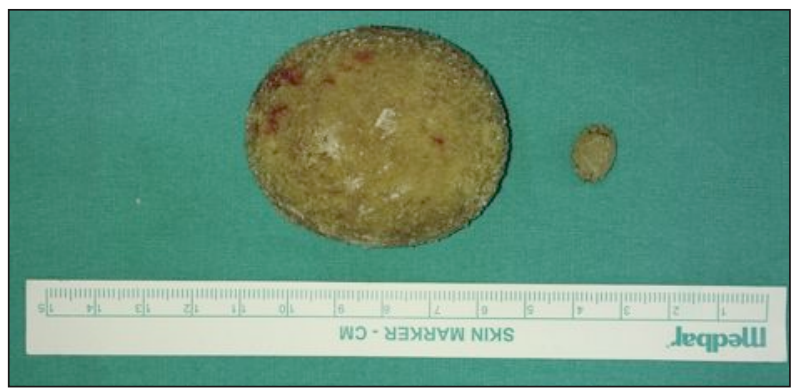

Cystoscopic examination and open procedure were done together on the same operative day. No anatomic urethral obstruction was observed and prostatic urethra and bladder neck were normal too. Cystoscopy normal demonstrated bilaterally normal ureteral orifices and a bladder stone which completely obstructed the bladder outlet as it was severely adherent to the bladder mucosa. The stone weighed $200 \mathrm{~g}$ and measured $5.0 \mathrm{~cm}$ x $6.0 \mathrm{~cm} \times 5.0 \mathrm{~cm}$ in size (Figure 3). Post-operative period was uneventful. The patient was discharged on fourth post-operative day and urethral catheter was removed on the seventh day. At the first month post-operative outpatient control, no residual bladder stone or ureterohydronephrosis was present.

\section{Discussion}

The presence of a huge bladder calculus is a rare cause of acute renal failure. According to the recent study from Komeya et al, it was reported that, according to English literature of the last several decades, only six papers reported acute renal failure caused by stones (4). The median age of patients was 62 years and the heavier weight was $200 \mathrm{~g}$. The level of serum creatinine on admission was higher than $672 \mu \mathrm{mol} / \mathrm{L}$ whereas in our patient was $848 \mu \mathrm{mol} / \mathrm{L}$. Nygaard et al reported that their patient's serum creatinine level was $1264 \mu \mathrm{mol} / \mathrm{L}$ on admission and the stone weighted $1640 \mathrm{~g}$ which was the largest stone ever removed by open cystolithotomy in literature(5). Additionally, in this patient an inoperable carcinoma of the bladder was detected more than four years later after cystolithotomy. In these papers, limited information was provided about the underlying disease which caused voiding disorder and bladder stones before admission. Patients usually complained recurrent urinary tract infection and long-term persistent urinary symptoms. Our patient had urinary tract infection on admission and we detected 100.000 E.Coli CFU/ml in urine culture that was treated by intravenous antibiotics before surgical operation.

\section{Conclusions}

In order to summarize about our case report, although single huge bladder stones rarely cause acute renal failure with respect to upper tract urinary stones, we should always remember this rare condition. We need to carefully consider two important aspects: 1) long-term urinary symptoms and recurrent urinary tract infections especially in men may cause huge bladder stone and acute renal failure 2) a 2 -fold increase in bladder cancer risk was observed in association with a history of bladder stones in a case-control study (6). Therefore the long delay in diagnosis of giant stones can cause squamous cell carcinoma of bladder. We should strictly follow-up patients with huge bladder stones for the risk of bladder cancer after operation.

\section{REFERENCES}

1. Childs MA, Mynderse LA, Rangel LJ, et al. Pathogenesis of bladder calculi in the presence of urinary stasis J Urol. 2013; 189:1347-1351.

2. Aydogdu O, Telli O, Burgu B, Beduk Y. Infravesical obstruction results as giant bladder calculi, Can Urol Asoc J. 2011; 5:77-78.

3. Hammad FT, Kaya M, Kazim E. Bladder calculi: did the clinical picture change? Urology 2006; 67:1154-8.

4. Komeya M, Sahoda T, Sugiura S, et al. A huge bladder calculus causing acute renal failure Urolithiasis 2012; 12:517-8.

5. Nygaard E, Terjesen T. Giant vesical calculas and anuria Scand J Urol Nephrol. 1976; 10:88-90.

6. Kantor AF, Hartge P, Hoover RN, et al. Urinary tract infection and risk of bladder cancer. Am J Epidemiol. 1984; 119:510-5.

\section{Correspondence}

Orcun Celik, MD (Corresponding Author)

orcuncelik82@hotmail.com

Tufan Suelozgen, MD

Salih Budak, MD

Yusuf Ozlem Ilbey, MD

Associate Professor

Endourology Section Urology Department, Tepecik Educational and Research Hospital

Gaziler Cd.No:468, Yenisehir - 35140, Izmir, Turkey 